\title{
EDITORIAL
}

\section{Interleukin-10, age and acute lung injury genetics: the action is in the interaction}

\author{
J.D. Christie
}

$\Delta$ cute lung injury (ALI) and its more severe form, acute respiratory distress syndrome (ARDS), are important public health issues, and both incidence and mortality increase with age [1]. Given that only a moderate percentage of individuals with the same level of exposure develop ALI/ ARDS, it is logical to investigate whether inherent interindividual variation will, in part, determine the risk of ALI/ARDS. Better understanding of the genetic factors influencing both risk of developing ALI/ARDS and outcomes following ALI/ ARDS may aid in the identification of high-risk individuals, suggest areas for laboratory research and lead to the development of more specific, targeted pharmacogenomic strategies for treatment and prevention [2].

Research on the genetic epidemiology of ALI/ARDS is in its early stages. Studies in this area have either used physiological hypotheses (including angiotensin-converting enzyme and surfactant proteins) [3,4] or bioinformatics approaches based on animal experiments to select candidate genes [5]. In this issue of the European Respiratory Journal, GONG et al. [6] provide an important contribution to understanding the genetic aetiology of ARDS and outcomes following ARDS. In a wellconstructed cohort study, the authors report an association of an interleukin (IL)-10 promoter polymorphism with ALI/ ARDS that differed by strata of age.

GoNG et al. [6] should be commended on several important strengths of their study. First, the large nested case-control design ensured that all non-ARDS subjects were drawn from the same at-risk cohort, minimising the bias due to selection of controls that can plague gene-association studies [7]. Secondly, rigorous methods for phenotyping ARDS were employed, thereby minimising misclassification. Thirdly, careful and rigorous statistical methods were used to test for interaction.

The resultant findings highlight some important and current issues in genetic studies of ALI/ARDS aetiology and outcomes. First, the main finding regarding ALI risk is an interaction of IL-10 genotype with age. The epidemiological term "interaction" refers to the concept that the effect of a risk factor (IL-10 genotype) on the outcome (ALI/ARDS) may be modified by a third factor (in this case age) [8]. Interactions are

CORRESPONDENCE: J.D. Christie, Division of Pulmonary, Allergy and Critical Care Medicine, Center for Clinical Epidemiology and Biostatistics, University of Pennsylvania School of Medicine, 423 Guardian Drive, 719 Blockley Hall, Philadelphia, PA 19104, USA. Fax: 1 2155735235. E-mail: jchristi@cceb.med.upenn.edu important to genetic studies of complex diseases, particularly in the case of ALI/ARDS where there are many variables that may modify disease risk and outcomes, such as different precipitating factors and comorbid diseases. The study of interactions in epidemiological studies is usually exploratory, unless specified a priori and the study is appropriately powered to detect them. In the study by GoNG et al. [6], the IL-10 promoter single nucleotide polymorphism (SNP) had an effect on the risk of ALI/ARDS only among subjects aged $>52$ yrs. While the authors should be commended for testing interactions using conservative statistical methods, given that this interaction was not hypothesised a priori, the results should be interpreted as exploratory and in need of confirmation. In addition, since the cut-off was driven by the data, and not a priori specified, further research should focus on solidifying the different effects of IL-10 across the broad spectrum of age to validate the cut-off age chosen.

Secondly, the authors focus on a single SNP in the promoter of a single gene. In candidate gene studies, both functional SNP studies and block haplotype-based approaches may provide useful and complementary information. Although the IL-10 -1082 SNP seems to have functional consequences, the results have been inconsistent in prior studies, as was acknowledged by GoNG et al. [6]. Prior studies of the IL-10 promoter region have evaluated several SNPs in the IL-10 promoter as a haplotype [9]; thus, the observed association may be due to other promoter SNPs or combinations thereof. Recent research has confirmed that much of genetic variation is inherited in blocks of single-stranded DNA, known as haplotype blocks $[10,11]$. In genetic epidemiology studies, haplotype-based approaches do not rely on functional assumptions and can use "haplotype-tagged" SNPs to efficiently test the association between block haplotypes and outcomes [12]. Available online databases, such as SeattleSNPs (http://pga.gs.washington. edu/gty_data/il10/), have defined tagged SNPs for the IL-10 gene in European-descent populations, thus facilitating these efforts [13]. Despite the importance of the study by GoNG et al. [6], future association studies of the IL-10 promoter region would also benefit from consideration of haplotype effects, using these and other tools [13].

Thirdly, the IL-10 SNP did not satisfy Hardy-Weinberg proportions in the non-ARDS (control) population; thus, the association of IL-10 with ALI/ARDS risk may have been affected by comparison with this control population, which had an abnormal distribution of alleles. The deviation from the expected Hardy-Weinberg proportions indicates that the 
proportion of alleles at the IL-10 -1082 site was not what one would expect in a general population at steady state. ARDS gene-association studies can be prone to "ascertainment bias" caused by the way that subjects are selected into the study, usually by admission to the intensive care unit (ICU). In the non-ARDS control population, the fact that the alleles of the IL$10-1082$ site were not present in expected proportions raises the point that the genotype may have affected entry into the ICU or mortality prior to admission to the ICU. Thus, the "protectiveness" of this SNP for mortality may actually be due to differential mortality prior to admission.

Despite these minor issues, the study by GoNG et al. [6] raises an important new set of questions for future studies. First, what is the role of IL-10 in ALI pathogenesis and resolution? Prior studies of IL-10 protein levels have been conflicting, in part due to fluctuations in levels during the course of critical illness, as well as interrelationships with other cytokines. Although true gene associations with disease are attractive because they probably do not represent epiphenomena, extrapolations of functional inference based on only one SNP in a single gene in a complex system may be limited [14]. Future studies of the IL-10 promoter should probably include other inflammatory genes to assess potential gene-gene interactions. Secondly, why would the IL-10 SNP have differential effects based on age? In addition to this observation being confirmed in other epidemiological studies, the results of the study by GONG et al. [6] imply that laboratory studies should include animals of different ages to investigate this phenomenon. Finally, the study by GoNG et al. [6] suggests that IL-10 is a good candidate gene to test as a potential predictor of ALI/ARDS risk and/or mortality for utility in risk stratification in clinical settings.

In summary, the study by GONG et al. [6] provides an important advance in our understanding of the genetics of acute lung injury/acute respiratory distress syndrome, and provides an important candidate gene for future study. Although future studies of acute lung injury/acute respiratory distress syndrome risk and outcomes may include more broad genome searches as technology advances, these results remind us that interactions with clinical variables will probably modify genetic associations with complex syndromes and, thus, must be accounted for in the design of genetic epidemiological studies. Furthermore, these results provide grounds for future research aimed at investigating the mechanisms underlying the link of variation in the interleukin-10 gene and acute lung injury/acute respiratory distress syndrome, as well as testing the relationship of genotype and age in studying acute lung injury/acute respiratory distress syndrome risks and outcomes.

\section{REFERENCES}

1 Rubenfeld GD, Caldwell E, Peabody E, et al. Incidence and outcomes of acute lung injury. N Engl J Med 2005; 353: 1685-1693.

2 Matthay MA, Zimmerman GA, Esmon C, et al. Future research directions in acute lung injury: summary of a National Heart, Lung, and Blood Institute working group. Am J Respir Crit Care Med 2003; 167: 1027-1035.

3 Gong MN, Wei Z, Xu LL, et al. Polymorphism in the surfactant protein-B gene, gender, and the risk of direct pulmonary injury and ARDS. Chest 2004; 125: 203-211.

4 Marshall RP, Webb S, Bellingan GJ, et al. Angiotensin converting enzyme insertion/deletion polymorphism is associated with susceptibility and outcome in acute respiratory distress syndrome. Am J Respir Crit Care Med 2002; 166: 646-650.

5 Ye SQ, Simon BA, Maloney JP, et al. Pre-B-cell colonyenhancing factor as a potential novel biomarker in acute lung injury. Am J Respir Crit Care Med 2005; 171: 361-370.

6 Gong MN, Thompson BT, Williams PL, et al. Interleukin-10 polymorphism in position -1082 and acute respiratory distress syndrome. Eur Respir J 2006; 27: 674-681.

7 Cardon LR, Palmer LJ. Population stratification and spurious allelic association. Lancet 2003; 361: 598-604.

8 Woodward M. Confounding and Interaction. In: Woodward M, ed. Epidemiology: Study Design and Analysis. New York, NY, USA, Chapman and Hall, 1999; pp. 145-189.

9 Lin MT, Storer B, Martin PJ, et al. Relation of an interleukin-10 promoter polymorphism to graft-versus-host disease and survival after hematopoietic-cell transplantation. N Engl J Med 2003; 349: 2201-2210.

10 McVean GA, Myers SR, Hunt S, Deloukas P, Bentley DR, Donnelly $\mathrm{P}$. The fine-scale structure of recombination rate variation in the human genome. Science 2004; 304: 581-584.

11 Phimister EG. Genomic cartography: presenting the HapMap. N Engl J Med 2005; 353: 1766-1768.

12 de Bakker PI, Yelensky R, Pe'er I, Gabriel SB, Daly MJ, Altshuler D. Efficiency and power in genetic association studies. Nat Genet 2005; 37: 1217-1223.

13 Altshuler D, Brooks LD, Chakravarti A, Collins FS, Daly MJ, Donnelly P. A haplotype map of the human genome. Nature 2005; 437: 1299-1320.

14 Cobb JP, O'Keefe GE. Injury research in the genomic era. Lancet 2004; 363: 2076-2083. 